\title{
Production of Donor-Derived Offspring by Transfer of Primordial Germ Cells in Japanese Quail
}

\author{
Tamao ONO, Takashi MATSUMOTO*, and Yukie ARISAWA** \\ Faculty of Agriculture, Shinshu University, Minamiminowa 399-4598, and Present address: \\ *Nankodo Co., Ltd, Tokyo 113-0033, Japan, **Department of Pathology and Laboratory \\ Medicine, University of Pennsylvania, Philadelphia, PA 19104-6082 U.S.A.
}

\begin{abstract}
We transfused concentrated primordial germ cells (PGCs) of the black strain (D: homozygous for the autosomal incomplete dominant gene, $D$ ) of quail into the embryos of the wild-type plumage strain (WP: $\left.d^{+} / d^{+}\right)$of quail. The recipient quail were raised until sexual maturity and a progeny test of the putative germline chimeras was performed to examine the donor gamete-derived offspring $\left(D / d^{+}\right)$. Thirty-one percent $(36 / 115)$ of the transfused quail hatched and 21 (13 females and 8 males) of them reached maturity. Five females and 2 males were germline chimeras producing donor gamete-derived offspring. Transmission rates of the donor derived gametes in the chimeric females and males were $1.8-8.3 \%$ and $2.6-63.0 \%$, respectively. Germline chimeric and the other putative chimeric males were also test-mated with females from the sex-linked imperfect albino strain (AL: $d^{+}$/ $d^{+}, \mathrm{al} / W$, where al indicates the sex-linked imperfect albino gene on the $Z$ chromosome, and $W$ indicates the $W$ chromosome) for autosexing of $W$-bearing spermatozoa: No albino offspring were born.
\end{abstract}

Key words: germline chimera, primordial germ cells, progeny test, transfusion, quail

\section{Introduction}

A number of attempts have been made to produce germline chimeras and donor gamete-derived offspring by the transfer of primordial germ cells (PGCs) in chickens $[6,12,13]$. This procedure will serve as a powerful tool for producing transgenic birds for genetic improvement and the production of useful products, and for preserving the foundation stocks of poultry [12]. Furthermore, it will be applied to maintain varieties of poultry which are endangered under natural conditions
[12].

PGCs are the first identifiable precursor cells for the gametes. Avian PGCs show a unique migration pathway during early development; at stages 11-16 [3], PGCs circulate in the bloodstream and by stages 20-24 they have migrated into the gonadal primordium, where they rapidly proliferate to form germ cells $[2,7,14]$. These features of PGCs' migration facilitate their isolation and transfer [16].

Recently, Yasuda et al. [16] developed a technique for concentrating PGCs collected from the embryonic

(Received 23 February 1998 / Accepted 6 May 1998)

Address corresponding: T. Ono, Laboratory of Developmental Biology, Faculty of Agriculture, Shinshu University, Minamiminowa 399-4598, Japan 
blood at stages $13-15$ by Ficoll density gradient centrifugation. With this technique, germline chimeric chickens were effectively produced by injecting concentrated PGCs isolated from the embryonic blood into the marginal vein of host embryos $[6,12]$.

In this study, we transfused concentrated PGCs of the black strain of quail into the embryos of the wildtype plumage strain of quail. The recipient embryos were raised until sexual maturity and a progeny test was performed to examine the donor gamete derived offspring.

\section{Materials and Methods}

Animals: Japanese quail (Coturnix japonica) of the black (D: homozygous for the autosomal incomplete dominant gene, $D$ ), the imperfect albino (AL: homozygous for the sex-linked recessive gene, $a l)$ and the wild-type plumage (WP: $d^{+} / d^{+}, A l^{+} / A l^{+}$and $d^{+} / d^{+}, A l^{+} /$ $W$, where $W$ indicates the $W$ chromosome) strains were used [10]. Fertilized eggs were collected daily and kept at $12^{\circ} \mathrm{C}$ for not more than 3 days. The eggs were then incubated in a commercial incubator at $37.5^{\circ} \mathrm{C}$ and $70 \%$ relative humidity with tilting to a 90 degree angle at $30 \mathrm{~min}$ intervals until the embryos reached stages 14-15.

Preparation of micropipettes: The micropipettes used for blood collection from donors (D) and injection to recipients (WP) were made from siliconized glass capillary tubes (G-1, Narishige, Tokyo) drawn out with a micropipette puller (PB-7, Narishige). The tips were beveled with a pipette grinder (EG-4, Narishige) at 20 degrees to an outer diameter of 30-80 $\mu \mathrm{m}$.

Preparation of PGCs: Blood was collected from the omphalomesenteric artery or the terminal sinus of $D$ embryos. The PGCs were separated from erythrocytes by Ficoll density gradient centrifugation [16]. Briefly, $100 \mu l$ of blood collected from approximately 50 eggs was pooled, dispersed in $500 \mu l$ of $10 \%$ foetal calf serum (FCS) in Leibovitz's L-15 medium and spun at $200 \mathrm{~g}$ for $5 \mathrm{~min}$. Pellets were then dispersed in $800 \mu \mathrm{l}$ of $15.5 \%$ Ficoll in L-15 and overlaid with $200 \mu l$ of 6.3\% Ficoll in calcium- and magnesium-free Dulbecco's phosphate-buffered saline. After centrifugation at 800 $\mathrm{g}$ for $30 \mathrm{~min}, 300-400 \mu \mathrm{l}$ of the PGC-rich fraction located in the layer between $15.5 \%$ and $6.3 \%$ Ficoll was transferred to a tube which contained $10 \%$ FCS in
L-15 and dispersed. Ficoll was removed by repeated centrifugation at $200 \mathrm{~g}$ for $5 \mathrm{~min}$. The concentration of the PGCs was calculated with a haemocytometer and a suspension of $100-200 \mathrm{PGCs} / \mu \mathrm{l}$ in $10 \% \mathrm{FCS}$ in L-15 was obtained.

Transfer of PGCs: The recipient WP embryos at stages 14-15 with egg yolk and albumen were transferred to $10-\mathrm{m} l$ plastic cups. Fine protein foam produced from $10 \%$ FCS in L-15 was pre-loaded into a micropipette, 1.0-1.5 $\mu l$ of PGC-suspension was transfused into the recipient terminal sinus and the perforation was sealed with the foam [16].

Embryo culture: After the transfusion, the recipient embryos were cultured in vitro until hatching in a standard culture system for quail embryos [8]. Briefly, the embryos in the plastic cups were transferred into bed shells prepared from the sharp halves of small hen's eggs, the shells were sealed with cling films (Saran Wrap) and thin hen's egg albumen as an adhesive. The embryos were then cultured at $37.5^{\circ} \mathrm{C}$ and $70 \%$ relative humidity with tilting to a 30 degree angle at $30 \mathrm{~min}$ intervals. Control embryos without PGC transfusion were also cultured until hatching.

Progeny test: The transfused quail were raised and a progeny test was carried out by a pair-mating with WP quail of the opposite sex. Donor gamete-derived offspring, namely heterozygous black $\left(D / d^{+}\right)$, were identified based on their feather color. Some transfused male quail were crossed with $\mathrm{AL}\left(d^{+} / d^{+}\right.$, al/W) females to demonstrate functional W-bearing spermatozoa. The resulting heterozygous black $\left(D / d^{+}, A L^{+} / a l\right.$ and $\left.D / d^{+}, A L^{+} / W\right)$ and albino $\left(D / d^{+}, a l / W\right.$, where $a l$ is epistastic to $D$ ) quail chicks were considered to be the donor gamete-derived offspring. If albino females $(D /$ $\left.d^{+}, a l / W\right)$ were produced, the W-chromosome involved would be derived from the $\mathrm{W}$-bearing spermatozoon which had originated in the ZW-bearing PGC.

\section{Results}

Among 115 embryos transfused with PGCs, 36 (31.3\%) hatched and 21 (13 females and 8 males) reached maturity. The hatchability of the control culture was $59.6 \%(34 / 57)$. The progeny test of the putative germline chimeras is shown in Table 1 . Of 1007 chicks born from the 13 putative germline chimeric females mated with WP males, 17 offspring were 
heterozygous black chicks born from 5 females. These 5 females were therefore considered to be germline chimeras in which $1.8-8.3 \%$ of the offspring were derived from the donor PGCs. Of the 8 putative germline chimeric males, 4 were crossed with WP females, and 13 heterozygous black chicks out of 311 were obtained. All the heterozygous chicks were derived from a single male (\#012), indicating that this male was a germline chimera with a transmission rate of $38.2 \%(13 / 34)$. This germline chimeric (\#012) and the other 4 putative germline chimeric males were also mated with AL females. Sixty-three percent (17/27) of the offspring derived from the gametes of \#012 were heterozygous black. Of the other 4 putative germline chimeras, one male was a germline chimera producing heterozygous black offspring with a transmission rate of $2.6 \%$ (4/ 153). No albino offspring were born.

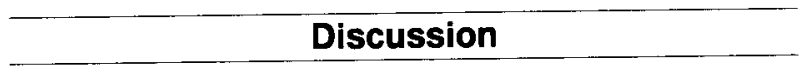

The hatchability of transfused embryos was over $30 \%$, and about $60 \%$ of the hatchlings were raised to maturity in the present study. Compared with the hatchabilities (17.9-28.2\%) of the PGC-transferred chickens reported by Naito et al. [6], that in the present study $(31.3 \%)$ was similar or somewhat better. The hatchability in the present study is satisfactory but the fall in hatchability with the PGC-treatment $(59.6 \%$ to $31.3 \%)$ is greater than that $(30.0-31.8 \%$ to $17.9-28.2 \%)$ reported by Naito et al. [6]. There is room for further practice and refinement of our technique for the introduction of PGCs. Naito et al. [6] injected chick PGCs into the bloodstream through the dorsal aorta of recipient chick embryos. They reported that it was simple to perform with no treatment needed to prevent hemor-

Table 1. Progeny test of putative germline chimeric quail ${ }^{\mathrm{a}}$

\begin{tabular}{lcccc}
\hline Sex and ID\# & $\begin{array}{c}\text { No.of } \\
\text { PGCs } \\
\text { transferred }\end{array}$ & $\begin{array}{c}\text { Strain of } \\
\text { mating } \\
\text { partner }\end{array}$ & $\begin{array}{c}\text { No. of } \\
\text { wild-type } \\
(d / d) \text { offspring }\end{array}$ & $\begin{array}{c}\text { No. of } \\
\text { heterozygous black } \\
(D / d) \text { offspring }\end{array}$ \\
\hline Female 001 & 200 & WP & 137 & $4(2.8 \%)$ \\
Female 005 & 100 & WP & 44 & $4(8.3 \%)$ \\
Female 008 & 150 & WP & 121 & $0(0.0 \%)$ \\
Female 009 & 150 & WP & 131 & $4(3.0 \%)$ \\
Female 010 & 200 & WP & 95 & $0(0.0 \%)$ \\
Female 014 & 200 & WP & 42 & $0(0.0 \%)$ \\
Female 015 & 200 & WP & 70 & $0(0.0 \%)$ \\
Female 016 & 200 & WP & 20 & $0(0.0 \%)$ \\
Female 103 & 100 & WP & 23 & $0(0.0 \%)$ \\
Female 104 & 200 & WP & 118 & $3(2.5 \%)$ \\
Female 105 & 150 & WP & 107 & $2(1.8 \%)$ \\
Female 106 & 125 & WP & 50 & $0(0.0 \%)$ \\
Female 108 & 125 & WP & 32 & $0(0.0 \%)$ \\
Male 003 & 300 & WP & 125 & $0(0.0 \%)$ \\
Male 007 & 100 & WP & 89 & $0(0.0 \%)$ \\
Male 011 & 200 & WP & 63 & $0(0.0 \%)$ \\
Male 012 & 200 & WP & 21 & $13(38.2 \%)$ \\
Male 012b & 200 & AL & 10 & $17(63.0 \%)$ \\
Male 013 & 200 & AL & 55 & $0(0.0 \%)$ \\
Male 101 & 100 & AL & 68 & $0(0.0 \%)$ \\
Male 102 & 200 & AL & 149 & $4(2.6 \%)$ \\
Male 107 & 150 & AL & 46 & $0(0.0 \%)$ \\
\hline Total & & & 1616 & $51(3.2 \%)$ \\
\hline
\end{tabular}

a: PGCs of the D strain were transferred to WP strain embryos; explanation of the strains are given in the text. b: ID\#012 was mated with both WP and AL quail for an interval of more than 1 month. c: No albino offspring were born. 
rhage and caused little damage to the recipient's development. They removed 4-10 $\mu \mathrm{l}$ of blood from the dorsal aorta of recipient chick embryos before the introduction of PGCs to reduce the population of the endogenous PGCs and to enhance the frequency of donor gamete-derived offspring. We tried to remove blood from the recipient quail embryos at first, but after several preliminary trials we decided not to remove blood in order to obtain better viability of embryos instead of enhanced frequency of donor gamete-derived offspring (unpublished observation). One germline chimeric male showed high transmission rates of donor-derived gametes of $38.2 \%$ and $63 \%$ when mated with WP and AL females, respectively. If the blood of the recipient could be removed prior to the PGC injection, the frequency of germline chimeras would be increased, yielding high transmission rates of the donor gamete. Germline chimeric quail have been produced by transfusing PGCs taken from the germinal crescent of quail embryos [15], but detailed information such as germline transmission rate, viability, etc., was not reported.

In the present study, the donor PGCs consisted of a mixed population of cells bearing $\mathrm{ZZ}$ and $\mathrm{ZW}$ sexchromosomes. Female germline chimeras have both endogenous and exogenous Z- and W-bearing ova. Sexing of the offspring was performed only for some of the heterozygous hatchlings in the present study, although both male and female offspring were born, indicating that both $\mathrm{W}$ - and Z-bearing donor gametederived ova were functional. By contrast, the male recipients received both $\mathrm{ZZ}$ - and unnatural $\mathrm{ZW}$-bearing PGCs. When the germline chimeric males were mated with WP females, both male and female offspring were born in the present study, although it was not determined whether the $Z$-bearing donor gamete-derived ova were from male ZZ- or female ZW-bearing PGCs. Unfortunately, no albino female offspring $\left(D / d^{+}, a l / W\right)$ were obtained from the test-mating between germline chimeric males and AL females. If albino females had been produced, it would have been direct evidence of the functional W-bearing spermatozoa. If the W-bearing spermatozoa were functional and fertilized W-bearing ova, the resulting zygotes would lack $\mathrm{Z}$ chromosomes and should be lethal [11]. In chickens, W-bearing spermatozoa were detected with fluorescent in situ and Southern hybridizations in a PGC-transferred rooster [11]. The population of $\mathrm{W}$-bearing spermato- zoa was quite low, indicating that most of the $\mathrm{W}$-bearing spermatozoa were impaired for some unknown reason. Abinawanto et al. [1] observed numerous spermatids and several spermatozoa bearing $\mathrm{W}$-chromosomes in the sex-reversed (genetically female and phenotypically male) chicken, indicating that the second meiosis occurred normally but that the transformation from the spermatid to the spermatozoon was partially impaired. Kagami et al. [4] injected a suspension of blastoderm cells from an individual chick embryo into another unincubated chick embryo in which the sex of the donor and recipient embryos was determined with a W-chromosome-specific DNA probe, and raised somatic and germline sex-chimeras. The female-derived Z-bearing spermatozoa fertilized ova and the male-derived Z-bearing ova were fertilized, and normal offspring were produced in both cases. On the other hand, the femalederived $\mathrm{W}$-bearing spermatozoa were unable to fertilize ova. In the testis, unnatural $\mathrm{ZW}$-bearing spermatogonia enter meiosis I and produce functional ZZ-bearing spermatocytes. The ZZ-bearing spermatocytes complete the second meiotic division, continue to differentiate during spermatogenesis, and leave the seminiferous tubules as functional spermatozoa. By contrast, the WW-bearing spermatocytes appear to have impaired spermiogenesis with a still unresolved defect [4].

In conclusion, the foreign quail PGCs that were transfused into the vascular system of quail embryos were capable of following a normal course of spermatogenesis or oogenesis and gave rise to functional spermatozoa or ova.

\section{Acknowledgments}

This study was supported in part by a Grant-in-Aid from the Ministry of Education, Science, Sports and Culture to T.O.

\section{References}

1. Abinawanto, Zhang, C., Saito, N., Matsuda, Y., and Shimada, K. 1998. Identification of sperm-bearing femalespecific chromosome in the sex-reversed chicken. J. Exp. Zool. 280: 65-72.

2. Fujimoto, T., Ukeshima, A., and Kiyofuji, R. 1976. The origin, migration and morphology of the primordial germ cells in the chick embryo. Anat. Rec. 185: 139-154.

3. Hamburger, V. and Hamilton, H.L. 1951. A series of normal stages in the development of the chick embryo. J. Morphol. 
88: 49-92.

4. Kagami, H., Clark M.E., Verrinder Gibbins, A.M., and Etches, R.J. 1995. Sexual differentiation of chimeric chickens containing $\mathrm{ZZ}$ and $\mathrm{ZW}$ cells in the germline. Mol. Reprod. Dev. 42: 379-387.

5. Kuwana, T. 1993. Migration of avian primordial germ cells toward the gonadal anlage. Develop. Growth \& Differ. 35: 237-243.

6. Naito, M., Tajima, A., Yasuda, Y., and Kuwana, T. 1994. Production of germline chimeric chickens, with high transmission rate of donor-derived gamates, produced by transfer of primordial germ cells. Mol. Reprod. Dev. 39: 153-161.

7. Nakamura, M., Kuwana, T., Miyayama, Y., and Fujimoto, T. 1988. Extragonadal distribution of primordial germ cells in the early chick embryo. Anat. Rec. 222: 90-94.

8. Ono. T., Murakami, T., Mochii, M., Agata, K., Kino, K., Otsuka, K., Ohta, M., Mizutani, M., Yoshida, M., and Eguchi, G. 1994. A complete culture system for avian transgenesis, supporting quail embryos from the singlecell stage to hatching. Dev. Biol. 161: 126-130.

9. Singh, R.P. and Meyer, D.B. 1967. Primordial germ cells in blood smears from chick embryos. Science 156: 1503 1504.

10. Somes, R. G., Jr. 1988. International Registry of Poultry
Genetic Stocks, Bulletin 476. Storrs Agricultural Experiment Station, University of Connecticut, Storrs, CT. 11. Tagami, T., Matsubara, Y., Hanada, H., and Naito, M. 1997. Differentiation of female chicken primordial germ cells into spermatozoa in male gonads. Develop. Growth Differ. 39: 267-271.

12. Tajima, A., Naito, M., Yasuda, Y., and Kuwana, T. 1993. Production of germ line chimera by transfer of primordial germ cells in the domestic chicken (Gallus domesticus). Theriogenology 40: 509-519.

13. Vick, L., Luke, G., and Simkiss, K. 1993. Germ-line chimaeras can produce both strains of fowl with high efficiency after partial sterilization. J. Reprod. Fert. 98: 637-641.

14. Watanabe, M., Naito, M., Sasaki, E., Sakurai, M., Kuwana, T., and Oishi, T. 1994. Liposome-mediated DNA transfer into chicken primordial germ cells in vivo. Mol. Reprod. Dev. 7FÉ 268-274.

15. Wentworth, B.C., Tsai, H., Hallett, J.H., Gonzales, D.S., and Rajcic-Spasojevic, G. 1989. Manipulation of avian cells and gonadal differentiation. Poultry Sci. 68: 999-1010.

16. Yasuda, Y., Tajima, A., Fujimoto, T., and Kuwana, T. 1992. A method to obtain avian germ-line chimeras using isolated primordial germ cells. J. Reprod. Fert. 96: 521-528. 